TITLE:

\title{
Sand structure construction in Uca lactea (De Haan, 1835) is related to tidal cycle but not to male or female densities
}

$\operatorname{AUTHOR}(S)$ :

Muramatsu, Daisuke

CITATION:

Muramatsu, Daisuke. Sand structure construction in Uca lactea (De Haan, 1835) is related to tidal cycle but not to male or female densities. Crustaceana 2010, 83(1): 29-37

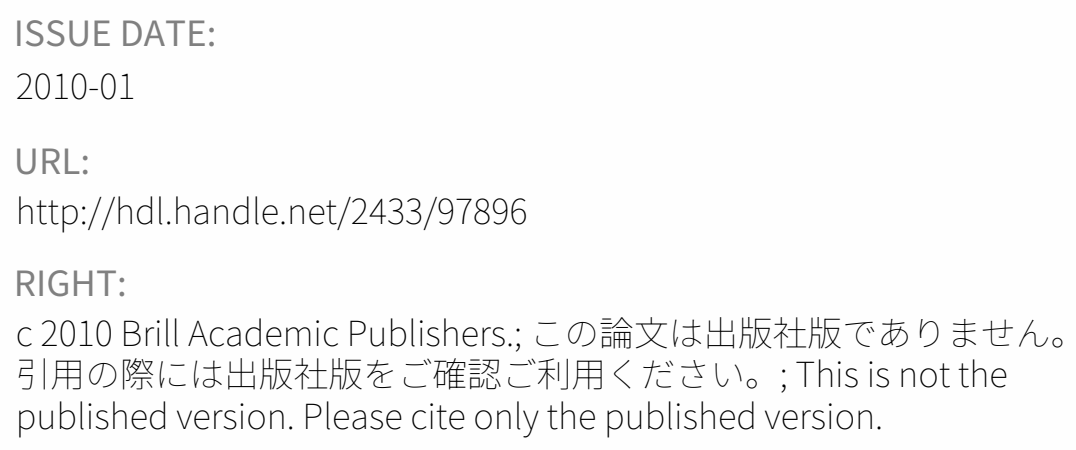


D. Muramatsu: Sand structures related to tidal cycle

\title{
SAND STRUCTURE CONSTRUCTION IN UCA LACTEA (DE HAAN, 1835) IS RELATED TO
}

\section{TIDAL CYCLE BUT NOT TO MALE OR FEMALE DENSITIES}

\author{
BY \\ DAISUKE MURAMATSU ${ }^{1}$ ) \\ Laboratory of Ethology, Graduate School of Science, Kyoto University, Sakyo-ku, Kyoto 606-8502, \\ Japan
}

\begin{abstract}
Males of several species of fiddler crabs construct sand structures at the entrance to their burrows to which they attract females for mating. Although sand structures are able to attract females, males do not always construct structures, and there is a considerable variation in the frequency of structure construction per day. To determine what factors cause the variation in the frequency of structure construction, I conducted a field observation on the structure construction of Uca lactea and examined the effects of air and soil temperatures, tidal amplitude, male and female densities, and the interaction between male and female densities. The frequency of structure construction was related to tidal amplitude. This may be a male response to the changing levels of female receptivity associated with the tidal cycle. The frequency of structure construction was also correlated positively with air temperature. Although courtship investment is expected to be influenced by the number of mates and rivals, neither male nor female density were significantly correlated with the frequency of structure construction.
\end{abstract}

${ }^{1}$ ) e-mail: muramatsu@ethol.zool.kyoto-u.ac.jp 


\section{INTRODUCTION}

Fiddler crabs (genus Uca) are a group of brachyuran crabs that live in burrows on intertidal mud- and sand-flats (Zeil et al., 2006). Males of at least 18 species of fiddler crabs construct sand structures at the entrance to their burrows (Christy \& Backwell, 2006). Sand structures are constructed by adult males in the breeding season (Yamaguchi, 1971, 2001a). In several fiddler crab species it has been shown that sand structures attract females for mating (Christy, 1988a, b; Christy et al., 2001, 2002, 2003a, b). Despite the mating benefit gained by possessing a structure, a considerable number of males do not construct structures in the breeding season (Yamaguchi, 1971; Zucker, 1974, 1978, 1981; Greenspan, 1982; Christy, 1988a, b; Backwell et al., 1995), and there is considerable variation in the frequency of structure construction per day. It is not clear why many males do not construct structures.

The variation in the frequency of structure construction may be due to the tidal cycle. Activities of fiddler crabs are confined to low tide periods when their habitat is exposed to the air (Pope, 2005), and the time and duration of habitat exposure change daily following the semilunar tidal cycle. Therefore, several environmental factors, such as light, temperature, and surface moisture, would change in a cycle. These environmental factors may affect the activity level of crabs (Crane, 1958) and thus influence structure construction.

Tidal amplitude itself may also influence the frequency of structure construction. It may be adaptive for females to modulate their sexual receptivity according to tidal amplitude in order to mate and release their larvae during the optimal timing of the semilunar tidal cycle (Christy, 1978, 2003; Morgan \& Christy, 1995). Males may adjust their courtship investment in structure construction according to tidal amplitude in response to the level of female receptivity.

The density of crabs on the mudflat surface sometimes affects the frequency of structure construction (e.g., Zucker, 1981). Perhaps more importantly, male and female densities can differently affect structure construction because investment in courtship may be influenced by the numbers of mates and rivals (see Kvarnemo et al., 1995; Shine et al., 2003).

Overall, there are many biotic and abiotic factors that may affect structure construction. In this study, I investigated the effects of these factors on structure construction under natural conditions in order to determine what influences the variation in the frequency of structure construction. Specifically, I examined the effects of air and soil temperatures, tidal amplitude, and male and female densities.

\section{MATERIAL AND METHODS}

All observations were carried out in a dense colony of Uca lactea (De Haan, 1835), which was approximately $3500 \mathrm{~m}^{2}$ in area, centred on an intertidal mudflat in the estuary of the Yabusa River in Ichiki-kushikino City, Kagoshima Prefecture, Japan (31은 $\left.41^{\prime} \mathrm{N} 130^{\circ} 17^{\prime} \mathrm{E}\right)$. There 
was no vegetation in this area. The crabs emerged from their burrows and were active on the mudflat surface during diurnal low tide; otherwise they stayed in their burrows and closed the entrance. Most of the structures were constructed by the time of the lowest tide. The entire study site was covered by the semidiurnal high tide, and all structures were destroyed by the tide. Before the habitat was covered by the incoming tide, crabs entered their burrows and plugged the entrances.

To investigate the density of sand structures, males, and females on the mudflat surface, five quadrats of $1 \mathrm{~m}^{2}$ each were established in the study site. These quadrats were established in line and spaced three $m$ from each other. The corners of the quadrats were marked with 1-m long, 4-mm diameter wooden poles, which were stuck vertically into the sediment so as to leave approximately $5 \mathrm{~cm}$ above the surface. Each day from 11 May until 6 September 2002, except for the days with heavy rain, I counted the number of burrow entrances and sand structures within the quadrats soon after the time of the diurnal lowest tide. I remained stationary beside each quadrat for a while to identify the resident of each burrow and recorded the sex of each resident. Crabs usually re-emerge from their burrow within a couple of minutes after disturbance. If residents did not appear within 10 minutes, the burrows were recorded as "Unknown sex".

Crabs were never touched during the observation and were sexed by their appearance based on the following criteria: presence of a major claw (only on males; see Yamaguchi, 1970), the colour of the left and right ambulatory legs (different colour in males, same colour in females; see Yamaguchi, 1971), and their feeding behaviour (males use one of a pair of chelipeds, whereas females use both chelipeds; see Yamaguchi, 2000). Crabs that were too small to be sexed (smaller than approximately $4 \mathrm{~mm}$ in carapace width) were disregarded. Males that had lost a major claw were also disregarded because they rarely construct structures (D. Muramatsu, unpubl. data). Air temperature approximately $1 \mathrm{~cm}$ above the mudflat surface in the shade and soil temperature approximately $10 \mathrm{~cm}$ into the substrate were taken at one quadrat soon after the time of the lowest tide.

To evaluate tidal amplitude I used the data of daily height of the daytime lowest tides. The height of the lowest tide is lower for spring tides than for neap tides because tidal amplitude is larger for spring tides. Tidal data were obtained from the Japan Meteorological Agency (http://www.jma.go.jp/jma/indexe.html), and the time was adjusted for the study site [by adding 25 minutes to the data at Kagoshima city ( $\left.\left.31^{\circ} 35^{\prime} \mathrm{N} 130^{\circ} 34^{\prime} \mathrm{E}\right)\right]$. To look for a seasonal trend in the frequency of structure construction I used the number of days from the first observation date as a variable.

The relationships between the frequency of structure construction and the density of males and females on the mudflat surface were analysed using generalized linear modelling techniques implemented in the statistical package $R$ ( $R$ Development Core Team, 2007). Generalized linear model can accept proportion data for the response variable, and $\mathrm{R}$ carries out weighted regression using individual sample sizes as weights. I fitted the frequency of structure 
construction (the number of burrows with a structure, vs. the number of burrows without a structure) as the response variable, which was weighted by daily sample size. As explanatory variables, I used the height of the lowest tide, days from the first observation date, air temperature, soil temperature, the number of males, the number of females, and the interaction between the number of males and that of females. The interaction between the two explanatory variables was the point-wise product of the two vectors, which was fitted as a new variable in the model (see Crawley, 2005). The data were tested for overdispersion by analysing residual deviance and the degrees of freedom using binomial errors and a logit link function. When overdispersion was present in the data, quasibinomial errors instead of binomial errors were used to compensate (see Crawley, 2005).

\section{RESULTS}

Sand structures were observed from 5 June until 21 August (fig. 1). During this period, the density of females was relatively low, especially from 26 June to 10 July and from 22 July to 3 August. The number of structures had a clear cycle, while male density remained relatively constant. Structures were constructed frequently on the days around new and full moon, i.e., the period of spring tides.

For the statistical analysis, I used the data from 22 June until 7 August, because males constructed few structures at the beginning and at the end of the period of structure construction (fig. 1). To analyse the data, I fitted quasibinomial errors for the model because the model was overdispersed. After model simplification by stepwise backwards elimination of the non-significant variables, a final model was constructed including only the height of the lowest tide and air temperature (table I). All other explanatory variables were rejected (table II).

\section{DISCUSSION}

The number of structures had a clear semilunar cycle in the present study. Activities of fiddler crabs are expected to be influenced by the semilunar tidal cycle because the time and duration of habitat exposure change daily following the tidal cycle. However, the number of active crabs on the mudflat surface did not show clear cycle in the present study. Therefore, tidal cycle influenced more strongly on the number of structures. During the period of sand structure construction, relatively few females were observed on the mudflat surface. This may be due to the incubation period of ovigerous females. During the period of egg incubation, females stay in the burrow and do not emerge on the mudflat surface until the larvae are released (Yamaguchi, 2001b). The number of females decreased twice during the period of structure construction in the 
present study. This is consistent with the reproductive cycle of females because females of Uca lactea have a lunar cycle in the reproduction and lay eggs twice during the breeding season (Yamaguchi, 2001a).

The frequency of structure construction in male $U$. lactea was related to the height of the lowest tide. This may have been caused by the changing levels of female receptivity due to the tidal cycle. Females benefit from releasing their larvae during large-amplitude tides because such tides rapidly transport larvae away from shorelines where planktivorous fishes abound (Morgan \& Christy, 1995). The average incubation period of $U$. lactea is 15.4 days (Yamaguchi, 2001b), which approximately equals one tidal cycle. Therefore, females that copulate during spring tides will be able to release their larvae during the next spring tide. Thus, to release their larvae during the large amplitude tides, females would attempt to copulate during spring tides. Males may follow the reproductive cycles of females and invest in structure construction according to tidal amplitude.

The activity levels of crabs are influenced by the external environment, such as light, tide, temperature, surface moisture, and rain (Crane, 1958). Especially, the thermal environment would be important for ectothermic animals, and many of their behaviours, include courtship, are influenced by temperature (Denoel et al., 2005). In the present study, there was a positive relationship between the frequency of structure construction and air temperature. Perhaps air temperature influenced the activity levels of males and consequently caused the variation of structure construction.

Female density did not affect the frequency of structure construction in the present study. This is not consistent with the contention that males increase their courtship investment when females abound. My study, however, focused on females that have their own burrows (= resident females) and did not include females that do not have their own burrows (= wandering females), which are also courted by males. Hence, the present study does not exclude the possibility that the frequency of structure construction is related to the density of wandering females. Males of $U$. lactea exhibit two types of mating tactics: surface mating and burrow mating. In the case of surface mating, males visit nearby resident females to copulate in the vicinity of the females' burrows, while in burrow mating, males wave their major claw to attract wandering females into their own burrows (Yamaguchi, 1971, 1972, 2001c; Murai et al., 1987; Goshima \& Murai, 1988). That fact that the density of resident females did not affect the frequency of structure construction in the present study indicates that the sand structures of $U$. lactea may not be related to surface mating. To test the relationship between structures and burrow mating, further investigations on wandering females will be needed.

Zucker (1981) suggested that structures constructed by U. musica terpsichores (Crane, 1941) and $U$. beebei (Crane, 1941) define male territories under conditions of high population density and reduce the frequency of combat among males. Other authors, however, have reported that structures have no territorial functions in several species of fiddler crabs (Christy, 1988a; Backwell et al., 1995; Christy et al., 2001). The present study also suggests non-territorial 
functions of structures in $U$. lactea because male density does not significantly affect the frequency of structure construction.

\section{ACKNOWLEDGEMENTS}

I would like to thank M. Imafuku and A. Mori for providing constructive comments on the manuscript. I also thank S. Cook and E. Nakajima for revising the English used in the manuscript. This research was financially supported in part by the Global COE Program A06 to Kyoto University.

\section{REFERENCES}

BackWell, P. R. Y., M. D. Jennions, J. H. Christy \& U. SchobeR, 1995. Pillar building in the fiddler crab Uca beebei: evidence for a condition-dependent ornament. Behav. Ecol. Sociobiol., 36: 185192.

CHRISTY, J. H., 1978. Adaptive significance of reproductive cycles in the fiddler crab Uca pugilator: a hypothesis. Science, New York, 199: 453-455.

- - 1988a. Pillar function in the fiddler crab Uca beebei (1): effects on male spacing and aggression. Ethology, 78: 53-71.

— - 1988b. Pillar function in the fiddler crab Uca beebei (2): competitive courtship signaling. Ethology, 78: 113-128.

- - 2003. Reproductive timing and larval dispersal of intertidal crabs: the predator avoidance hypothesis. Rev. Chilena. Hist. nat., 76: 177-185.

CHRISTY, J. H. \& P. R. Y. BACKWELL, 2006. No preference for exaggerated courtship signals in a sensory trap. Anim. Behav., 71: 1239-1246.

Christy, J. H., P. R. Y. BAckWell \& S. Goshima, 2001. The design and production of a sexual signal: hoods and hood building by male fiddler crabs Uca musica. Behaviour, 138: 1065-1083.

Christy, J. H., P. R. Y. Backwell, S. Goshima \& T. Kreutera, 2002. Sexual selection for structure building by courting male fiddler crabs: an experimental study of behavioral mechanisms. Behav. 
Ecol., 13: 366-374.

CHRISTY, J. H., P. R. Y. BACKWELL \& U. SCHOBER, 2003a. Interspecific attractiveness of structures built by courting male fiddler crabs: experimental evidence of a sensory trap. Behav. Ecol. Sociobiol., 53: 84-91.

Christy, J. H., J. K. Baum \& P. R. Y. Backwell, 2003b. Attractiveness of sand hoods built by courting male fiddler crabs, Uca musica: test of a sensory trap hypothesis. Anim. Behav., 66: 8994.

Crawley, M. J., 2005. Proportion data. In: Statistics: an introduction using R. 247-262. (John Wiley \& Sons, West Sussex).

CRANE, J., 1958. Aspects of social behavior in fiddler crabs, with special reference to Uca maracoani (Latreille). Zoologica, 43: 113-130.

Denoel, M., M. Mathieu \& P. Poncin, 2005. Effect of water temperature on the courtship behavior of the Alpine newt Triturus alpestris . Behav. Ecol. Sociobiol., 58: 121-127.

GoSHIMA, S. \& M. MURAI, 1988. Mating investment of male fiddler crabs, Uca lactea. Anim. Behav., 36: 1249-1251.

GREENSPAN, B. N., 1982. Semi-monthly reproduction cycles in male and female fiddler crab, Uca pugnax. Anim. Behav., 30: 1084-1092.

Kvarnemo, C., E. Forsgren \& C. Magnhagen, 1995. Effects of sex ratio on intra- and inter-sexual behaviour in sand gobies. Anim. Behav., 50: 1455-1461.

MORGAN, S. G. \& J. H. CHRISTY, 1995. Adaptive significance of the timing of larval release by crabs. Am. Nat., 145: 457-479.

MuRAI, M., S. Goshima \& Y. HenMI, 1987. Analysis of the mating system of the fiddler crab, Uca lactea. Anim. Behav., 35: 1334-1342.

POPE, D. S., 2005. Waving in a crowd: fiddler crabs signal in networks. In: P.K. McGregor (ed.), Animal communication networks. 252-276. (Cambridge University Press, Cambridge).

R Development Core Team, 2007. R: A language and environment for statistical computing. (R 
Foundation for Statistical Computing, Vienna).

SHINE, R., T. LANGKILDE \& R. T. MASON, 2003. The opportunistic serpent: Male garter snakes adjust courtship tactics to mating opportunities. Behaviour, 140: 1509-1526.

YamaGuCHI, T., 1970. Ecology of a fiddler crab (Uca lactea) (1). Calanus, 2: 5-30.

— - 1971. Courtship behavior of a fiddler crab, Uca lactea. Kumamoto J. Sci. Biol., 10: 13-37.

- - 1972. Ecology of a fiddler crab (Uca lactea), (2) Courtship and mating behaviors. Calanus, 3: $38-53$.

- - 2000. Sexual differences in feeding by the fiddler crab, Uca lactea (De Haan). Crustac. Res., 29: 121-132.

- - 2001a. The breeding period of the fiddler crab, Uca lactea (Decapoda, Brachyura, Ocypodidae). Crustaceana, 74: 285-293.

- - 2001b. Incubation of eggs and embryonic developmentof the Fiddler crab, Uca lactea (Decapoda, Brachyura, Ocypodidae). Crustaceana, 74: 449-458.

- - 2001c. The mating system of the fiddler crab, Uca lactea (Decapoda, Brachyura, Ocypodidae). Crustaceana, 74: 389-399.

ZeIL, J., J. M. Hemmi \& P. R. Y. BACKWELL, 2006. Fiddler crabs. Curr. Biol., 16: 40-41.

ZUCKER, N., 1974. Shelter building as a means of reducing territory size in the fiddler crab, Uca terpsichores (Crustacea: Ocypodidae). Am. Midl. Nat., 91: 224-236.

— - 1978. Monthly reproductive cycles in three sympatric hood-building tropical fiddler crabs (genus Uca). Biol. Bull., 155: 410-424.

- -1981 . The role of hood-building in defining territories and limiting combat in fiddler crabs. Anim. Behav., 29: 387-395. 
Table I

Generalized Linear Model relating the height of the lowest tide and air temperature to the frequency of males that constructed structures in Uca lactea (De Haan)

\begin{tabular}{lcccccc}
\hline \hline Model term & $\underline{\mathrm{N}}$ & $\underline{\mathrm{df}}$ & Estimate & $\mathrm{SE}$ & $\underline{\mathrm{t}}$ & $\underline{\mathrm{p}}$ \\
\hline Intercept & & & -2.184 & 1.219 & -1.791 & 0.082 \\
Lowest tide & 38 & 1 & -0.008 & 0.002 & -3.791 & 0.001 \\
Air temp. & 38 & 1 & 0.081 & 0.040 & 2.041 & 0.049
\end{tabular}

The model is quasibinomial with a logit link; the dispersion parameter for quasibinomial family is 3.319 . Null deviance is 203.32 on 37 degrees of freedom and residual deviance is 124.54 on 35 degrees of freedom. The $p$-values are rounded to three decimal places. 
Table II

Rejected terms of Generalized Linear Models resulting from model simplification in Uca lactea (De Haan)

\begin{tabular}{|c|c|c|c|c|c|c|c|c|c|}
\hline Rejected term & $\underline{N}$ & Estimate & SE & $\begin{array}{l}\text { Change } \\
\text { deviance }\end{array}$ & in & $\begin{array}{l}\text { Residual } \\
\text { deviance }\end{array}$ & $\underline{\mathrm{df}}$ & $F$ & $p$ \\
\hline Male*Female & 38 & 0.001 & 0.006 & 0.093 & & 107.941 & 1,31 & 0.028 & 0.869 \\
\hline Soil temp. & 38 & -0.038 & 0.141 & 0.233 & & 108.174 & 1,32 & 0.072 & 0.791 \\
\hline Male density & 38 & -0.012 & 0.018 & 1.366 & & 109.54 & 1,33 & 0.435 & 0.514 \\
\hline Days & 38 & -0.012 & 0.007 & 8.482 & & 118.022 & 1,34 & 2.724 & 0.108 \\
\hline Female density & 38 & 0.025 & 0.018 & 6.519 & & 124.541 & 1,35 & 2.025 & 0.164 \\
\hline
\end{tabular}

Estimates and SEs shown are the values when each term was fitted last in the model. The significance of terms was assessed by the changes in deviance when the term was removed from the final model, using the F-test. "Male*Female" indicates the interaction between male and female densities. "Days" indicates the days from the first observation date. 


\section{FIGURE CAPTION}

Fig. 1. Daily fluctuation patterns of the numbers of males, females, and structures of Uca lactea (De Haan, 1835) as well as those of environmental factors.

For "Male", "Female", "Structure", and "Unknown sex" the mean value of five quadrats of $1 \mathrm{~m}^{2}$ each is shown. "Lowest tide" indicates the height of the diurnal lowest tide. 


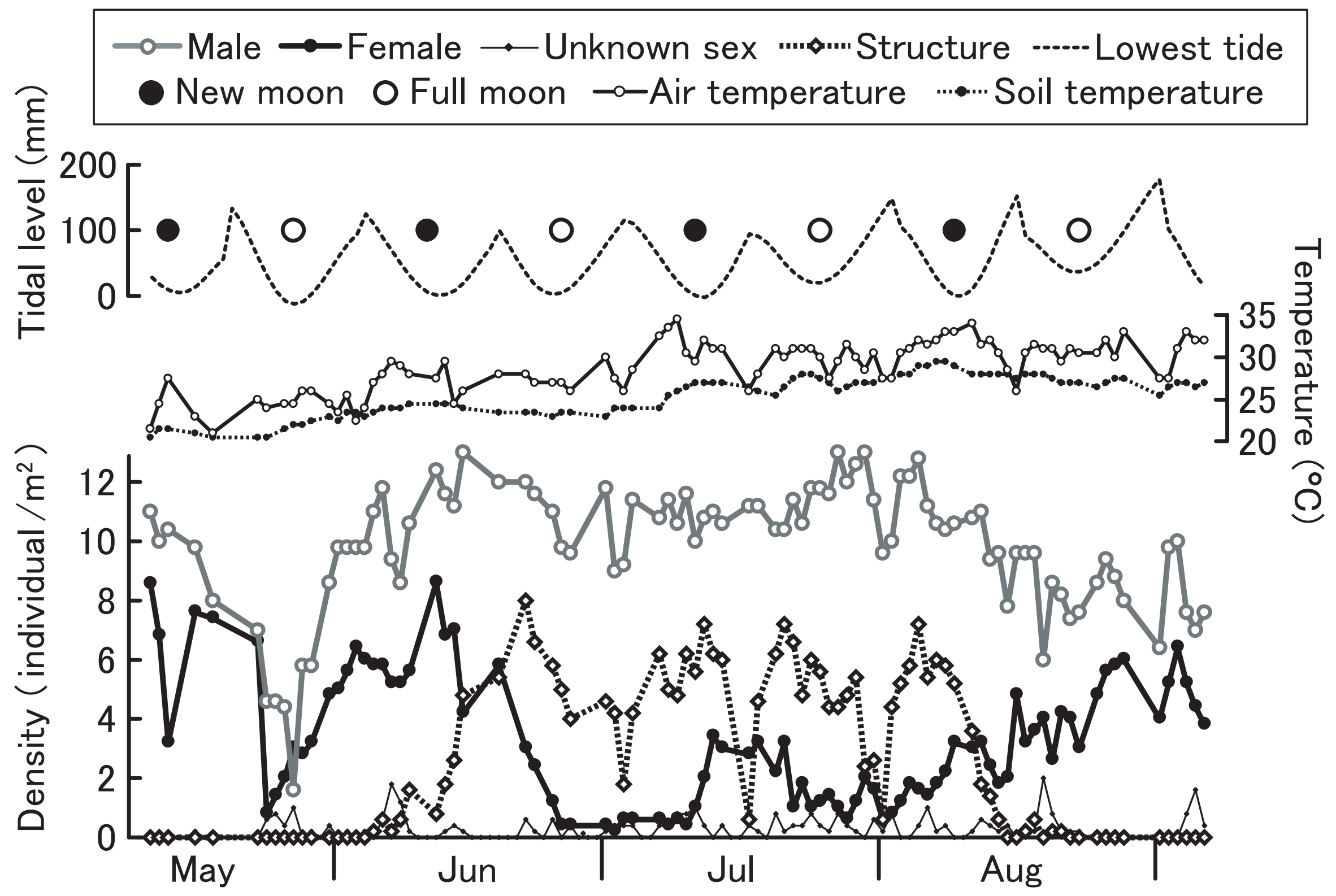

Fig. 1 